\title{
INFRAHYOID FLAP RECONSTRUCTION OF ORAL CAVITY AND OROPHARYNGEAL DEFECTS IN ELDERLY PATIENTS WITH SEVERE GENERAL COMORBIDITIES
}

\author{
Alberto Deganello, MD, PhD, ${ }^{1}$ Gianni Gitti, MD, PhD, ${ }^{1}$ Giampiero Parrinello, MD, ${ }^{1}$ \\ Guglielmo Larotonda, MD, ${ }^{1}$ Giuseppe Meccariello, MD, ${ }^{1}$ C. René Leemans, MD, PhD, ${ }^{2}$ \\ Oreste Gallo, MD' \\ ${ }^{1} 1$ st Clinic of Otolaryngology/Head and Neck Surgery, Department of Surgical Sciences, University of Florence, Florence, \\ Italy. E-mail: adeganello@hotmail.com \\ ${ }^{2}$ Department of Otolaryngology-Head and Neck Surgery, VU University Medical Center, Amsterdam, The Netherlands
}

Accepted 11 July 2011

Published online in Wiley Online Library (wileyonlinelibrary.com). DOI: 10.1002/hed.21913

\begin{abstract}
Background. In current practice, surgeons frequently deal with elderly patients who have severe medical comorbidities.

Methods. We present our series of 18 consecutive patients with severe general comorbidities that received infrahyoid flap reconstruction. The results were compared with those of 16 consecutive patients in good general medical state receiving free radial forearm flap reconstruction during the same study period. We also describe an original method for tongue base reconstruction using the infrahyoid flap.

Results. No total flap necrosis was experienced; successful separation between oral cavity/oropharyngeal contents and neck spaces was obtained in all patients with a low rate of general complications. No significant differences were found with regard to verbal intelligibility and diet scores between groups.

Conclusions. Infrahyoid flap in high-risk cases represents a valid alternative to free radial forearm flap. We introduced a novel technical innovation for tongue base reconstruction using the infrahyoid flap with very encouraging results. ○ 2011 Wiley Periodicals, Inc. Head Neck 00: 000-000, 2011
\end{abstract}

Keywords: free radial forearm flap; infrahyoid flap; oral cavity reconstruction; oropharyngeal reconstruction; high-risk patients

The application of microvascular free flaps is the most widespread method currently used for the reconstruction of extensive defects after resection of head and neck cancer because of their versatility and reliability. The success rate of free tissue transfers has risen to $>95 \%$, and the free radial forearm flap (FRFF) together with the free anterolateral thigh flap are currently considered the gold standard for soft tissue reconstruction of oral cavity and oropharyngeal defects. ${ }^{1,2}$

Correspondence to: A. Deganello

๑ㄱ 2011 Wiley Periodicals, Inc.
In current practice, surgeons frequently deal with elderly patients who have severe medical comorbidities and pretreated patients with recurrent disease or second primary malignancies. There are no agreed-upon universally validated contraindications for microvascular reconstruction in head and neck surgery; the trend in recently published reports is to extend indications for free flaps even in generally compromised patients and in the vessel depleted neck. $^{3,4}$

Furthermore, free flap reconstruction also seems to be reliable in elderly patients, ${ }^{5,6}$ despite the risk that general comorbidities, especially diabetes mellitus, pose to the success of microvascular transfers. ${ }^{7}$

However, not all patients are ideal candidates for free flap reconstruction, and not every defect strictly requires a free flap transfer to achieve good functional results. Thus there is a need for comparable alternatives. The infrahyoid flap (IHF) in head and neck reconstruction was first described by Wang et al. ${ }^{8,9}$ The major blood supply of this pedicled flap is derived from the superior thyroid artery; all its branches, except the posterior branch to the thyroid gland, have tiny tributaries entering the infrahyoid muscles and the overlying skin. Therefore, the flap can be harvested as a fasciomyocutaneous ${ }^{10,11}$ or a myofascial flap. ${ }^{12,13}$ It has proven its reliability and good functional results in various sites of head and neck reconstruction, especially for oral tongue and base of tongue reconstruction. ${ }^{8-14}$

At our institution, FRFF remains the first choice for soft tissue reconstruction of oral cavity and oropharyngeal defects that present a communication with neck spaces. However, instead of performing free flap reconstructions in cases that are considered to be unsuitable or suboptimal for microvascular procedures, alternative pedicled flaps are considered. Although the temporal flap and pectoralis major flap 


\begin{tabular}{|c|c|c|}
\hline Score & Diet & Speech \\
\hline 1 & $\begin{array}{l}\text { Regular diet without } \\
\text { restrictions }\end{array}$ & Always understandable \\
\hline 2 & Moist or soft diet & $\begin{array}{l}\text { Usually understandable, but } \\
\text { with frequent repetition or } \\
\text { face-to-face contact required }\end{array}$ \\
\hline 3 & Liquid diet & $\begin{array}{l}\text { Difficult to understand, even with } \\
\text { face-to-face contact }\end{array}$ \\
\hline 4 & $\begin{array}{l}\text { Tube-dependent } \\
\text { intake }\end{array}$ & $\begin{array}{l}\text { Never understandable, with } \\
\text { written communication required }\end{array}$ \\
\hline
\end{tabular}

represent the alternatives in case of unfavorable anatomic conditions (vessel depleted neck and previous chemoradiation), the IHF represents our first alternative to FRFF in high-risk patients because of severe general comorbidities. We present our results in 18 consecutive patients with severe general comorbidities that received IHF reconstruction as alternative to FRFF reconstruction. We also describe an original method for tongue base reconstruction using the infrahyoid fasciomyocutaneous flap. We critically compared healing and functional results between this series of 18 high-risk patients with a population of 16 subjects in good general medical condition who received FRFF reconstruction during the same study period.

\section{MATERIALS AND METHODS}

Data Collection. We reviewed the medical records of 88 consecutive patients who had free flap or pedicled flap reconstruction, performed by the first author (A.D.), at the department of Otolaryngology-Head and Neck Surgery of the University of Florence, Italy, between July 2006 to May 2010.

Follow-up data were obtained in all patients using clinical chart notes. Disease was staged in accord with the 6th edition of the TNM classification established by the Union Internationale Contre le Cancer/ American Joint Committee on Cancer (UICC/AJCC) ${ }^{15}$ using all the information available, including physical findings, imaging studies, and pathology reports.

The preoperative medical status of each patient was assessed by the anesthesiologists using the American Society of Anesthesiologists (ASA) classification of physical status.

Follow-up data including status of the flap and complications were collected. Postoperative functional results regarding diet and speech were assessed by a physician at outpatient follow-up consultation with use of a score system from 1 to 4 (Table 1).

Patients. From the 88 consecutive head and neck reconstructions, we identified 68 cases in which the defect of the oral cavity or oropharynx was in communication with neck spaces as a result of transmandibular or pull-through approaches. The reconstruction was accomplished with free radial forearm flap in 16 patients, infrahyoid flap in 18 patients, pectoralis major flap in 16 patients, temporal myofascial flap in 10 patients, fibula osteocutaneous flap in 5 patients, rectus abdominis flap in 2 patients, and latissimus dorsi in 1 patient.

We compared results between 18 patients who had IHF reconstruction (group 1, G1) and 16 patients in good general conditions that received FRFF reconstruction (group 2, G2).

G1 accounted for 12 men and 6 women, 12 receiving IHF for oral cavity and 6 for oropharyngeal reconstruction. All flaps were harvested from the same neck side of the primary tumor during homolateral neck dissection; 10 patients had bilateral neck dissection. For flap harvesting technique we refer to our previous report. $^{10}$

The mean age in G1 was 69.6 years (range, 55-83 years; median, 72 years); 3 patients were classified ASA II, the remaining ASA III. The mean dimensions of the skin paddle of the IHF were $6.5 \mathrm{~cm} \times 3.5 \mathrm{~cm}$ (mean surface area, $22.7 \mathrm{~cm}^{2}$ ). Contraindications for FRFF reconstruction in G2 were: severe comorbidities (diffuse atherosclerosis, diabetes mellitus, heart failure) in 15 cases, and age exceeding 80 years with moderate comorbidities in 3 cases.

G2 accounted for 12 men and 4 women; 9 patients received a FRFF to reconstruct a defect of the oral cavity, whereas 7 patients had a reconstruction of the oropharynx. The mean age in G2 was 58.2 years (range, 45-70 years; median, 58 years) and all patients were classified ASA I-II. The mean dimensions of the skin paddle of the FRFF were $7.1 \mathrm{~cm} \times$ $6.3 \mathrm{~cm}$ (mean surface area, $44.7 \mathrm{~cm}^{2}$ ). In all cases end-to-end arterial anastomoses were performed between the facial and radial arteries.

In 11 cases a single venous anastomosis was performed, whereas in 5 cases a double venous drainage was provided. In all cases the main recipient vessel was the internal jugular vein. In 2 cases anastomoses were performed on the contralateral side of the primary tumor.

Between groups we recorded and compared flap viability, operative time, blood loss and blood transfusion, postoperative intensive care unit (ICU) recovery, postoperative complications, postoperative reinterventions, duration of hospitalization, hospital readmissions related to head and neck surgery within 6 months, oral intake restoration time, time of tracheotomy closure, diet, and speech assessment.

Statistical Analysis. Statistical analysis was performed with an IBM computer (International Business Machines Corp., Armonk, NY) using STATA (Stata Corp., College Station, TX). Differences in mean values between groups were tested with Wilcoxon-Mann-Whitney test; for categorical variables, 


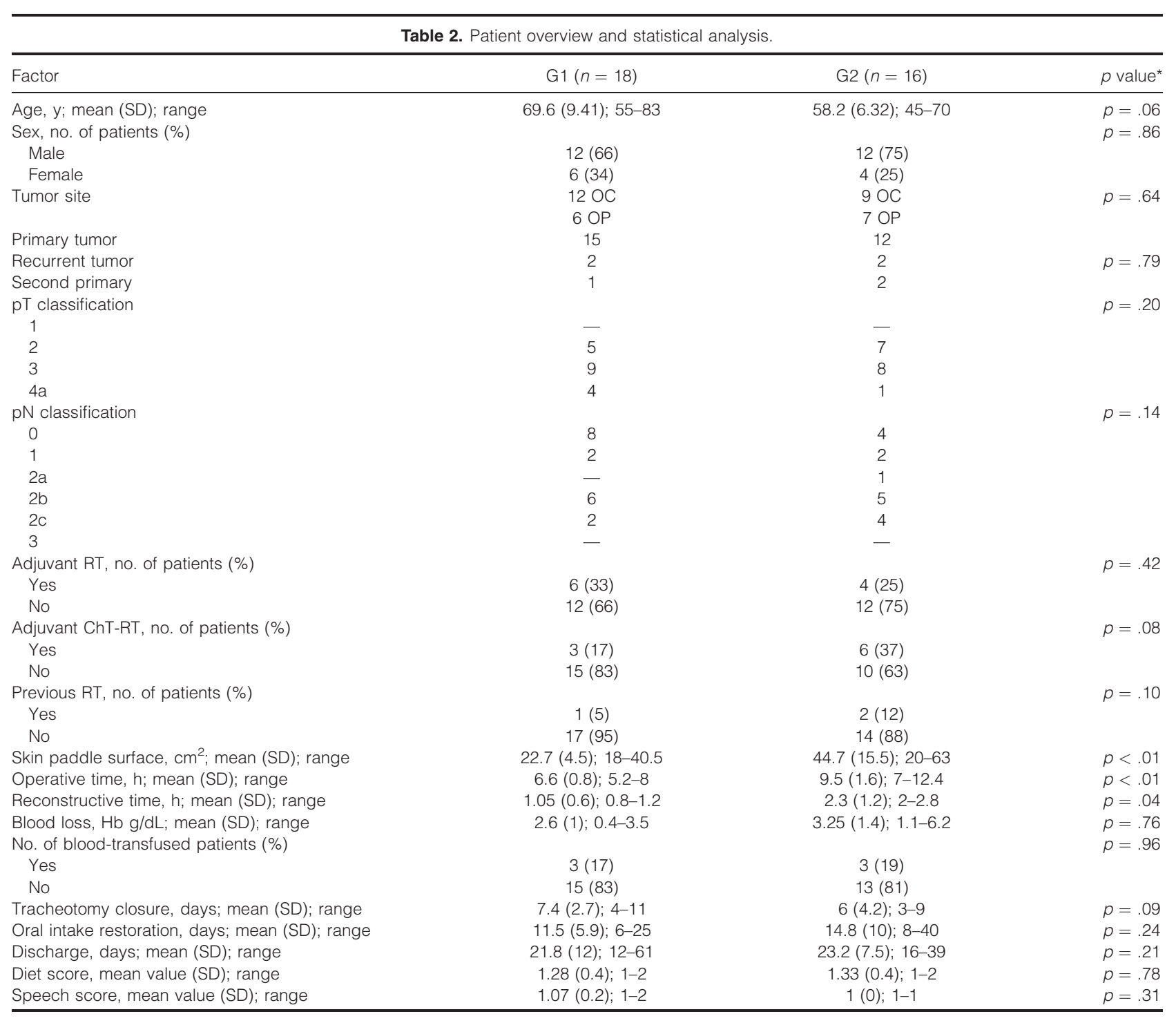

Abbreviations: OC, oral cavity; OP, oropharynx; RT, radiotherapy; ChT, chemotherapy; SD, standard deviation; Hb, hemoglobin.

*Differences in mean values between groups were tested with Wilcoxon-Mann-Whitney test; for categorical variables, the Pearson's chi-square test was used.

the Pearson chi-square test was used: probability values $<0.05$ were considered statistically significant.

\section{RESULTS}

Patient characteristics and results are displayed and summarized in Table 2.

The mean operative time in G1 was 6 hours 40 minutes (range, 5 hours 20 minutes to 8 hours), whereas in G2 it was 9 hours (range, 7 hours to 12 hours 40 minutes).

Postoperative intensive care recovery was used in 4 patients in G1 with a mean stay of 3 days and in 4 G2 patients with a mean stay of 3.7 days.

Flap Survival. No total flap necrosis was experienced in the series; successful separation between oral cavity/oropharyngeal contents and neck spaces was obtained in all patients.

In $\mathrm{G} 1,1$ patient developed a venous congestion revealed by the color of the skin paddle. Superficial cuts were made on the flap and heparin solution was injected twice a day; after 1 week the necrotic skin was removed, revealing underlying healthy muscles. Complete reepithelization occurred within 3 weeks (see Figure 1).

In G2, 1 patient required postoperative revision of the venous anastomosis 8 hours after the end of surgery; intraluminal thrombus was found and removed at the end-to-side confluence between the cephalic vein and the preserved caudal stump of the internal jugular vein. The flap reconstructed the lateral oropharyngeal wall and was double folded to restore half the soft palate. After microvascular revision the flap slowly developed marginal necrosis on its upper distal 


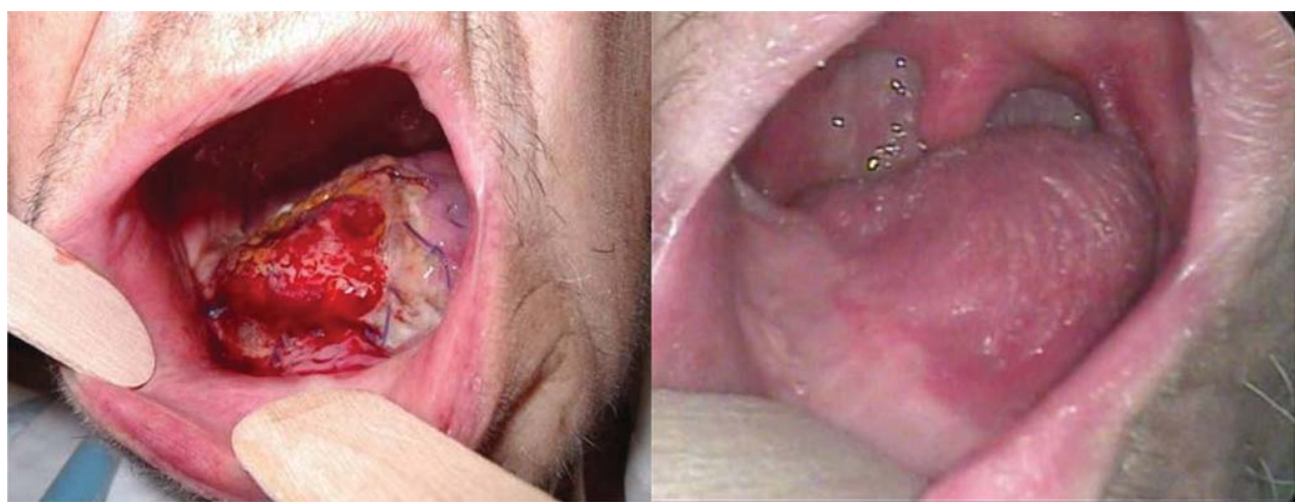

FIGURE 1. An 83-year-old woman with T4aNO oral tongue squamous cell carcinoma treated with pull-through resection encompassing three fourths of the mobile tongue, "en bloc" modified radical neck dissection, infrahyoid flap reconstruction. Complete reepithelization after superficial skin necrosis. [Color figure can be viewed in the online issue, which is available at wileyonlinelibrary.com.]

third. Further reconstruction of the soft palate using the remaining uvula under local anesthesia was required to prevent open rhinolalia and nasal regurgitation.

No patient was readmitted within 6 months.

Complications. The overall rate of complications was $14.7 \%$ (5/34), including partial necrosis $(2 / 34,1$ in G1 and 1 in G2) and postoperative pneumonia (3/34, 1 in G1 and 2 in G2). The rate of complications that required surgical revision was $2.9 \%$ (1/34). Indication for surgical revision was venous congestion and subsequent marginal necrosis in $1 \mathrm{G} 2$ case. The remaining complications were successfully treated with conservative management.

Functional Results. All patients were discharged with complete restoration of oral intake (mean time, 14 days; range, 7-18 days) and tracheotomy closure (mean time, 6 days; range, 3-10 days). Mean discharge time after surgery was 22 days (range, 12-37 days) with no differences between groups (21.8 days G1 and 23.2 days G2). No significant differences were found with regard to verbal intelligibility and diet score between groups.

\section{DISCUSSION}

Reconstruction of the oral cavity and oropharyngeal defects requires a thoughtful approach to guarantee a safe healing process and to enhance residual functionality. In the present study, we analyzed reconstructions performed by a single surgeon (A.D.) to avoid interoperator differences and we focused on soft tissue reconstructions to test different options. We selected only defects in communication with neck spaces to represent a similar level of complexity in these reconstructions. In fact, transoral resections are mostly performed for small tumors, where the reconstruction in these cases is less difficult, using primary closure, local flaps, or skin grafts only.
Because voluntary dynamic reconstruction is not currently achievable, optimal reconstructive outcome would be aimed at enhancing residual function and allowing good mobility of the preserved structures around the resected area. The replacement of dynamic structures with static ones has obvious limitations so that a thoughtful analysis of the anticipated defect and impairment is mandatory.

In our series FRFF appeared to be an excellent reconstructive method, confirming all advantages that make it the most popular and widespread microvascular flap in head and neck reconstruction.

The long pedicle allowed anastomoses to be performed in the contralateral neck side in 2 cases. We experienced a single case of venous congestion that was solved with microvascular revision. The problem was caused by a displacement of the caudal remaining stump of the internal jugular vein that had been superiorly fixed to prevent collapse and to facilitate venous drainage from the flap and from the middle thyroid vein. Refixation of the venous stump together with the removal of the intraluminal thrombus solved the problem. In this situation, however, extravenous anastomosis between 1 comitant vein and the external jugular system might have overcome venous congestion.

Although age itself is not a major risk factor, the mortality and morbidity rates for major surgical procedures are definitely higher in the elderly population compared with younger adults. ${ }^{16}$ The death rate resulting from surgery increases 3 -fold each year after the age of $60 .{ }^{17}$ This risk is more significant after age 70 and patients aged 80 years or older are more prone to operative mortality and morbidity. ${ }^{18}$ In a study of 78 patients aged 70 years or older that underwent free flap transfer for head and neck reconstruction, Coskunfirat et $\mathrm{al}^{19}$ reported an overall success rate of $96 \%$; however, postoperative medical complications arose in $44.1 \%$ of ASA III patients and in $50 \%$ of ASA IV.

IHF represented an excellent alternative solution to FRFF in high-risk populations in terms of healing 


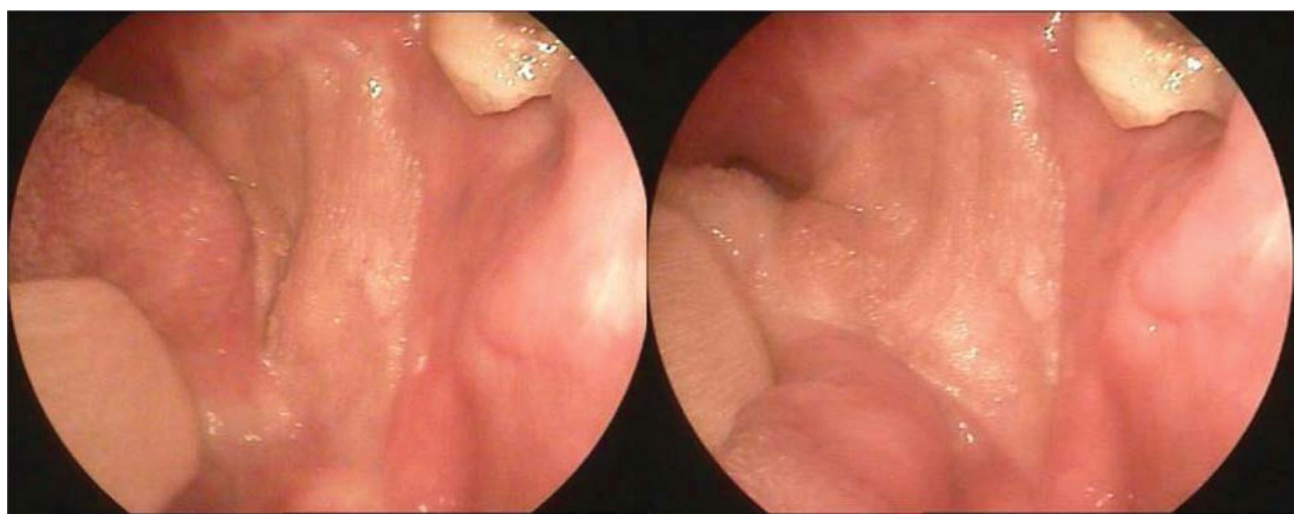

FIGURE 2. Infrahyoid flap reconstruction of the left retromolar trigone, anterior pharyngeal pillar, and posterior third of the tongue. [Color figure can be viewed in the online issue, which is available at wileyonlinelibrary.com.]

and functional results. Average age in G1 was 11 years higher than that in G2; patients presented severe comorbidities with $83.3 \%(15 / 18)$ assessed as being ASA III. Despite this, all patients had a successful reconstruction, with excellent functional results and a low rate of general complications. In 1 elderly patient (83 years old) with diabetes mellitus, we experienced superficial skin necrosis but healthy muscles provided complete reepithelization without scar fixation of the residual tongue (see Figure 1). In microvascular reconstructions the hemorheologic status of the patient must be carefully controlled during the early postoperative period to achieve good results; the maintenance of optimal parameters is particularly difficult in weak patients suffering from severe general comorbidities. ${ }^{20-22}$ It is also recognized that the risk for thrombosis is highest during the first 2 postoperative days. ${ }^{23,24}$ For prevention, several agents have been used such as heparin, acetylsalicylic acid, dextran, and prostaglandin E1. Side effects, other than bleeding risk of anticoagulation, should not be underestimated. Aspirin can cause gastric ulceration and nephrotoxicity. Heparin can cause heparine- induced thrombocytopenia. Dextran is known for anaphylaxis, pulmonary and cerebral edema, and platelet dysfunction. ${ }^{25}$ Conversely, in pedicled flap reconstruction this aspect is not as crucial as it is for microvascular procedures.

The majority of myocutaneous flaps for head and neck reconstruction (eg, pectoralis major, trapezius, latissimus dorsi) are quite bulky; conversely, the IHF is thin and pliable. Although the latter is not as thin and pliable as FRFF, it appears to be extremely suitable for all sites within the oral cavity and oropharynx, preventing salivary fistulas in the neck and allowing good motility of the tongue (Figures 2 and 3 ).

In cases of tongue reconstruction our practice is to always preserve the motor innervations of the infrahyoid muscles (provided by the ansa cervicalis) to prevent subsequent atrophy, as originally suggested by Wang et al. ${ }^{9}$ Conversely, for other sites, denervation atrophy of the underlying muscles will increase the flap's plasticity and pliability.

Furthermore, we are pleased to highlight a new personal modification of the surgical technique for base of tongue reconstruction that was used in this

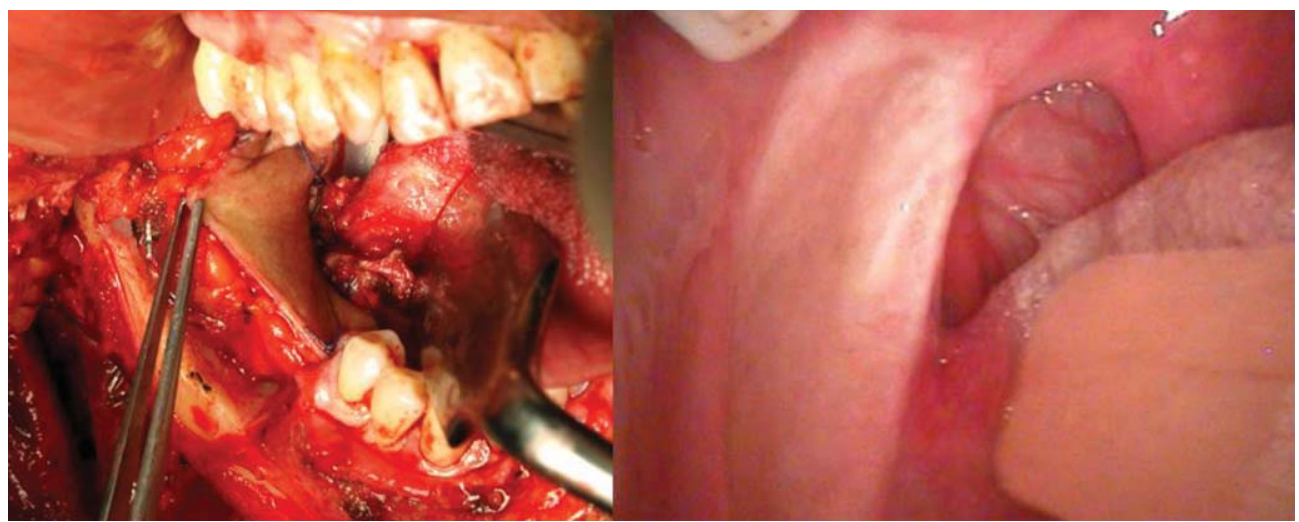

FIGURE 3. Infrahyoid flap reconstruction of the right tonsillar region, soft palate, and retromolar trigone. [Color figure can be viewed in the online issue, which is available at wileyonlinelibrary.com.] 

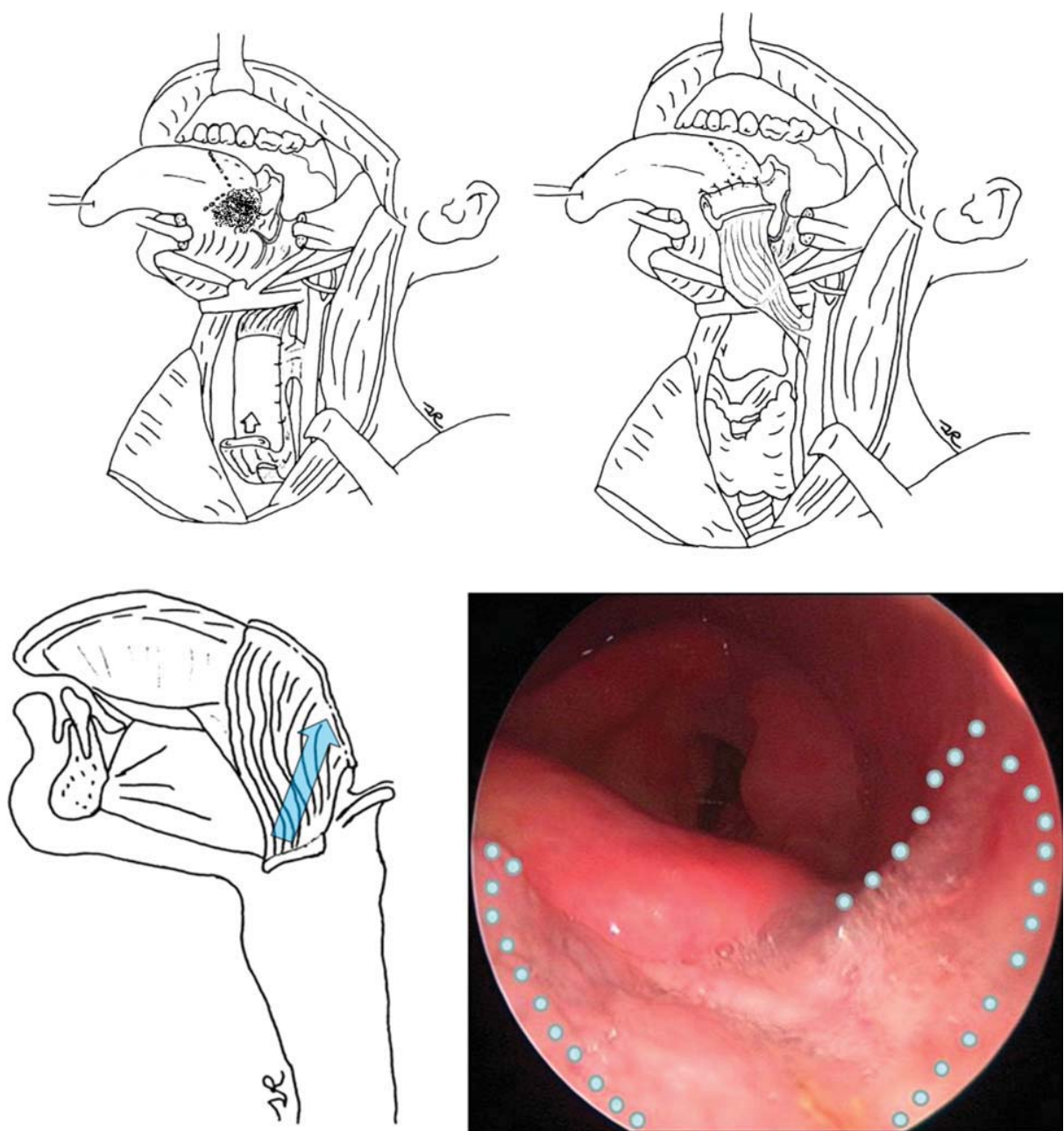

FIGURE 4. Base of tongue reconstruction after transmandibular resection of pT3N1 SCC at the left hand side. Infrahyoid flap (dotted line) has been transposed maintaining the muscular insertions at the hyoid bone. During deglutition, the hyoid bone elevates and pushes the flap backward, so helping with bolus propulsion. [Color figure can be viewed in the online issue, which is available at wileyonlinelibrary.com.]

series. We noted that, in cases of tongue base reconstruction, the transposition of the flap without detaching it from the hyoid bone (that acts as rotational pivot), improves swallowing efficacy. In fact, during deglutition, the hyoid bone elevates and pushes the flap backward, thus helping with bolus propulsion (as shown by dynamic fibroscopic investigations). For defects limited to the tongue base, IHF is perfectly suited to the resected area having the desired thickness. For all the above-mentioned reasons IHF is becoming our preferred method for base of tongue reconstructions (see Figure 4). In this series we used this technique in 4 patients with very promising results; however, our purpose was to further validate the technique in the future with more cases and videofluoroscopic studies. The reconstruction of oral tongue and base of tongue with infrahyoid flap has also been developed by a German group that has used a myofascial transposition of the flap. Whenever the resection encompassed more than half of the oral tongue and/or base of tongue, the infrahyoid myofascial flap was lined with a microvascular FRFF. ${ }^{12,13}$ However, in the description of surgical technique contained in their reports, the flap is being systematically transected from the hyoid bone; furthermore, the authors do not harvest the skin attached to the infrahyoid muscle group with the aim of reducing operative time for the surgical procedure and postoperative donor site morbidity.

In our series IHF reconstruction proved to be quick and convenient: the average operative reconstructive time in G1 was 1 hour and 25 minutes less than it was in G2; furthermore, in G1 only 1 surgical team was needed. The flap was harvested after neck dissection without interfering with oncologic radicality and all donor sites were primary closed with good aesthetic results (see Figure 5). The mean reconstructed surface area was $22.7 \mathrm{~cm}^{2}$, making this flap 


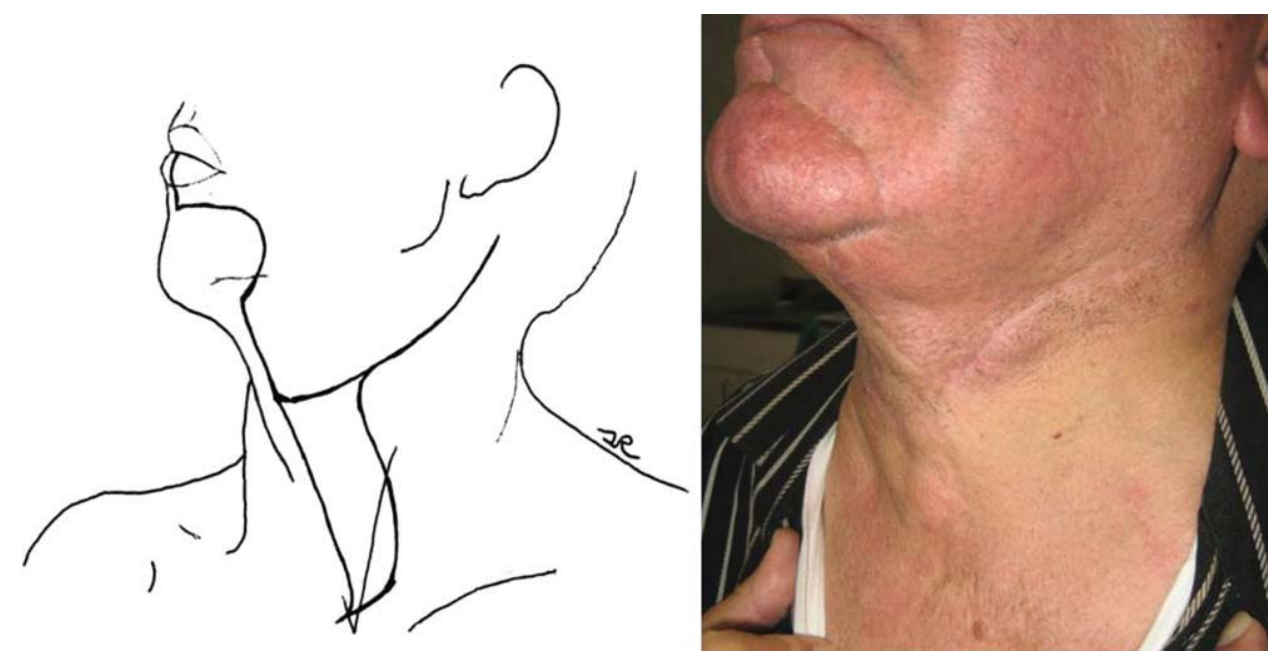

FIGURE 5. Donor site result following primary closure. [Color figure can be viewed in the online issue, which is available at wileyonlinelibrary.com.]

particularly suitable for medium-sized defects; the almost double surface of the skin paddle for the FRFF is mainly explained by its higher pliability. In fact, this thin fasciocutaneous flap offers a much more tailored reconstruction, providing an effective lining for all recesses of the resected area, and occasionally it can also be double folded ( 2 cases in this series). Conversely, the IHF is mainly used to connect the mucosal edges of the resection, whereas all recesses will be covered by the infrahyoid muscles. Nevertheless, for larger defects, IHFs with skin paddles measuring $9 \times 4.5 \mathrm{~cm}\left(40.5 \mathrm{~cm}^{2}\right)$ and $7.5 \times 4 \mathrm{~cm}$ $\left(30 \mathrm{~cm}^{2}\right)$ have been easily transposed in this series.

Disadvantages of IHF mainly coincide with its contraindications: previous thyroid surgery or neck dissection, N3 neck metastasis, and positive lymph nodes at level III-IV. This flap is also better not harvested in previously irradiated necks. All these contraindications pose consistent limitations to the use of this reconstructive option. The IHF must always be planned in advance and cannot represent a back-up solution in case of other flap failure, since it cannot be used in a previously operated neck. In fact, probable damages to the superior thyroid artery and/or vein and/or possible elevation of the skin overlying the strap muscles prevent the possibility of relying on this myocutaneous flap. Previous radiotherapy is not an absolute contraindication, but preoperative careful evaluation of the intended skin paddle is recommended: if lack of pliability, radiation-induced fibrosis, and/or telangectasias are encountered, then a decrease in blood supply to the skin through the perforator vessels is probably occurring and the flap is better not harvested. However, if these features are lacking and the appearance of the skin is normal, then the flap can be considered. In this series we used it in 1 postradiation neck without facing postoperative complications.

\section{CONCLUSIONS}

In our recent experience, FRFF still remains the firstchoice flap for many oral cavity and oropharyngeal soft tissue reconstructions. IHF in high-risk cases represents a valid alternative with excellent functional results. IHF does not require a second surgical team, change of the patient's position during surgery, or sophisticated harvesting procedures. Furthermore, we introduced a novel technical innovation for tongue base reconstruction using the IHF so that it has become our preferred method for this specific area and we are now using it as first choice rather than FRFF.

Acknowledgment. The authors thank Sabino Russo, MD, for surgical illustrations.

\section{REFERENCES}

1. Suh JD, Sercarz JA, Abemayor E, et al. Analysis of outcome and complications in 400 cases of microvascular head and neck reconstruction. Arch Otolaryngol Head Neck Surg 2004;130:962-966.

2. Novak CB, Lipa JE, Noria S, Allison K, Neligan PC, Gilbert RW. Comparison of anterolateral thigh and radial forearm free flap donor site morbidity. Microsurgery 2007;27:651-654.

3. Jacobson AS, Eloy JA, Park E, Roman B, Genden EM. Vesseldepleted neck: techniques for achieving microvascular reconstruction. Head Neck 2008;30:201-207.

4. Hanasono MM, Barnea Y, Skoracki RJ. Microvascular surgery in the previously operated and irradiated neck. Microsurgery 2009;29:1-7.

5. Beausang ES, Ang EE, Lipa JE, et al. Microvascular free tissue transfer in elderly patients: the Toronto experience. Head Neck 2003;25:549-553.

6. Nao EE, Dassonville O, Chamorey E, et al. Head and neck freeflap reconstruction in the elderly. Eur Ann Otorhinolaryngol Head Neck Dis 2011;128:47-51.

7. Joo YH, Sun DI, Park JO, Cho KJ, Kim MS. Risk factors of free flap compromise in 247 cases of microvascular head and neck reconstruction: a single surgeon's experience. Eur Arch Otorhinolaryngol 2010;267:1629-1633.

8. Wang HS, Shen JW. Preliminary report on a new approach to the reconstruction of the tongue. Acta Acad Med Prim Shanghai 1980;7:256-259. 
9. Wang HS, Shen JW, Ma DB, Wang JD, Tian AL. The infrahyoid myocutaneous flap for reconstruction after resection of head and neck cancer. Cancer 1986;57:663-668.

10. Deganello A, Manciocco V, Dolivet G, Leemans CR, Spriano G. Infrahyoid fascio-myocutaneous flap as an alternative to free radial forearm flap in head and neck reconstruction. Head Neck 2007;29:285-291.

11. Dolivet G, Gangloff P, Sarini J, et al. Modification of the infra hyoid musculo-cutaneous flap. Eur J Surg Oncol 2005;31:294298.

12. Remmert SM, Sommer KD, Majocco AM, Weerda HG. The neurovascular infrahyoid muscle flap: a new method for tongue reconstruction. Plast Reconstr Surg 1997;99:613-618.

13. Windfuhr JP, Remmert S. Infrahyoid myofascial flap for tongue reconstruction. Eur Arch Otorhinolaryngol 2006;263:1013-1022.

14. Minni A, Mascelli A, Suriano M. The infrahyoid myocutaneous flap in intra-oral reconstruction as an alternative to free flaps. Acta Otolaryngol 2010;130:733-738.

15. Sobin LH, Wittekind Ch, editors. TNM classification of malignant tumors, 6th ed. International Union Against Cancer. New York: Wiley-Liss; 2002.

16. Cole WH. Medical differences between young and the aged. Am Geriatr Soc 1970;18:589-614.

17. Variakojis RJ, Roizen MF.Preoperative evaluation of the elderly. In: McLeskey $\mathrm{CH}$, editor. Geriatric anesthesiology.Baltimore: Williams \& Wilkins; 1997. pp 165-185.
18. Ziffren SE, Hartford CE. Comparative mortality for various surgical operations in older versus younger age groups. J Am Geriatr Soc 1972;20:485-489.

19. Coskunfirat OK, Chen HC, Spanio S, Tang YB. The safety of microvascular free tissue transfer in the elderly population. Plast Reconstr Surg 2005;115:771-775.

20. Guarnieri T, Filburn CR, Zitnik G, Roth GS, Lakatta EG. Contractile and biochemical correlates of beta-adrenergic stimulation of the aged heart. Am J Physiol Heart Physiol 1980;239:H501H508.

21. Mannino DM, Davis KJ. Lung function decline and outcomes in an elderly population. Thorax 2006;61:472-477.

22. Jones NF, Jarrahy R, Song JI, Kaufman MR, Markowitz B. Postoperative medical complications (not microsurgical complications) negatively influence the morbidity, mortality, and true costs after microsurgical reconstruction for head and neck cancer. Plast Reconstr Surg. 2007;119:2053-2060.

23. Brown JS, Devine JC, Magennis P, Sillifant P, Rogers SN, Vaughan ED. Factors that influence the outcome of salvage in free tissue transfer. Br J Oral Maxillofac Surg 2003;41:16-20.

24. Kroll SS, Schusterman MA, Reece GP, et al. Timing of pedicle thrombosis and flap loss after free-tissue transfer. Plast Reconstr Surg 1996;98:1230-1233.

25. Kruse AL, Luebbers HT, Grätz KW, Obwegeser JA. Factors influencing survival of free-flap in reconstruction for cancer of the head and neck: a literature review. Microsurgery 2010;30:242-248. 